\title{
25 Research Square \\ Investigate the Double Peaks in Main Emission of UVB LEDs
}

\section{Tsung-Yen Liu}

National Taiwan University

Shih-Ming Huang

Chang Gung Memorial Hospital

Mu-Jen Lai

jiangxi Litkconn Academy of Optical Research

Rui-Sen Liu

Jiangxi Litkconn Academy of Optical Research

Chieh-Hsiung Kuan

National Taiwan University

Lin Ray-Ming ( $\nabla$ rmlin@mail.cgu.edu.tw)

Chang Gung University https://orcid.org/0000-0002-6919-4742

\section{Nano Express}

Keywords: ultraviolet, light-emitting diodes (LED), AlGaN, exciton.

Posted Date: July 14th, 2021

DOI: https://doi.org/10.21203/rs.3.rs-680585/v1

License: (c) (i) This work is licensed under a Creative Commons Attribution 4.0 International License.

Read Full License 


\section{Abstract}

In this study we suppressed the parasitic emission caused by electron overflow found in typical UVB lightemitting diodes (LEDs). Furthermore, modulation of the p-layer structure and doping profile allowed us to decrease the relaxation time of the holes to reach conditions of quasi-charge neutrality in the UVB quantum well. Our UVB LED (sample A) exhibited a clear exciton emission, with its peak near $306 \mathrm{~nm}$ and a band-to-band emission at $303 \mathrm{~nm}$. The relative intensity of the exciton emission of sample A decreased as a result of a thermal energy effect. At temperatures of up to $363 \mathrm{~K}$, sample A displayed the exciton emission. Our corresponding UVC LED (sample B) exhibited only a Gaussian peak emission at a wavelength of approximately $272 \mathrm{~nm}$.

\section{Introduction}

Compact high-efficiency solid-state ultraviolet (UV) light sources, including light-emitting diodes (LEDs) and laser diodes, are of considerable technological interest for use as alternatives to large, toxic, lowefficiency gas lasers and mercury lamps. In addition, mercury lamps are common sources of UV radiation, particularly at shorter wavelengths, and they are bulky and require high voltages for operation. LEDs as replacements would be attractive in terms of their smaller size and lower operating voltages. [1] There are several definitions of UV bands depending on the concern, application, or industry. For health and safety purposes, UV light is commonly divided into UVA (400-320 nm), UVB (320-280 nm), and UVC $(280-100 \mathrm{~nm})$ bands. [2,3] UV light can have damaging effects on biological systems, particularly at shorter wavelengths. Indeed, many sterilizations and food processing systems use UVC light. On the other hand, small amounts of UVB can be therapeutic for certain skin conditions (e.g., psoriasis; optimum wavelength: ca. $311 \mathrm{~nm}$ ). In this paper, we report our attempts to use electron/hole pairs bound in excitons as a light source for AIGaN-based LEDs. Introducing this emission, resulting from an exciton transition, has the potential to provide exciton-related light-emitting devices to replace gas light sources with solid-state light sources.

A large exciton binding energy induces strong excitonic effects in AIGaN. For example, the optical absorption spectrum exhibits sharp resonance features [4-6], due to dominating direct transitions between the valence and conduction bands at the same K (wave vector) points. [7-9] These strong excitonic effects cause a significant transfer of oscillator strength from the band-to-band transition to the 1s exciton state. [10] The ratio of the oscillator strength of the 1s exciton state to the band-to-band transitions can reach up to 100-fold. [11-13] AIN (exciton binding energy: $80 \mathrm{meV}$ ) is not the only material in which excitons have been used as the basis of light-emitting devices. Several other wide-bandgap semiconductors, including diamond (exciton binding energy: ca. $80 \mathrm{meV}$ ) and zinc oxide (exciton binding energy: $60 \mathrm{meV}$ ), have stable excitons at room temperature. [1] The binding energy for stable excitons must be greater than the equivalent thermal energy at room temperature. The dominant forces binding excitons are the coulombic interactions between the negative and positive charge carriers. There are many factors restricting electrons and holes from forming excitons. For example, the $\mathrm{K}$ values of the 
bottom of the conduction band and the top of the valence band. If there are too many electrons nearby, however, they will block the coulombic attraction between the electron and the hole required to form the exciton. Generally, exciton emission is observed from relatively pure materials, but not from highly doped materials. [7]

The exciton binding energy is a measure of the stability of the electron/hole pair. An exciton with high binding energy is less likely to be scattered by lattice vibrations to form free charge carriers, or to undergo conversion to free charge carriers as a result of the presence of impurities or defects. Consequently, an exciton having a high binding energy will have a lifetime longer than that of one with a weak binding energy. The higher exciton binding energy also increases the radiative rate and decreases the nonradiative rate. [14] These attributes are important to consider when developing photonic devices. Diamond's exciton, having a binding energy of approximately $80 \mathrm{meV}$, is stable at room temperature; sharp emission lines at $235 \mathrm{~nm}(5.27 \mathrm{eV})$ and $242 \mathrm{~nm}(5.12 \mathrm{eV})$ have been attributed to free excitons. [1] Koizumi et al. were the first to report a diamond-based device emitting in the deep-UV; a forward bias of $20 \mathrm{~V}$ produced a "strong" UV emission at $235 \mathrm{~nm}$, even though we might rule out diamond as a light emitter because of its indirect band gap. [15] Zinc oxide is another potential exciton light source; its binding energy is approximately $60 \mathrm{meV}$. Excitation behavior was observed in the UVA band at $390 \mathrm{~nm}$ upon irradiating ZnO with 355-nm light from an Nd:YAG solid-state laser, suggesting the possibility of developing a $\mathrm{ZnO}$ laser having a low threshold current. Nevertheless, n-type $\mathrm{ZnO}$ is relatively easy to prepare, but p-type $\mathrm{ZnO}$ is difficult to realize. If a reliable method was available for creating p-type $\mathrm{ZnO}$, ZnO-based devices would presumably have distinct advantages over current commercial GaN LEDs and laser diodes. [16] Taniyasu et al. reported LED wavelengths that were decreased down to $210 \mathrm{~nm}$; they prepared a PIN diode having an electroluminescence wavelength of $210 \mathrm{~nm}$-very close to that of free exciton recombination in undoped AIN in photoluminescence measurements. [17] The AIN exciton has a large binding energy of approximately $80 \mathrm{meV}$, imparting stability at room temperature. Because of its large activation energy (630 meV), the doping efficiency of Mg-doped p-AIN is very low. [18] Furthermore, no conduction band discontinuity was available to enhance carrier confinement at the AIN homojunction. The output power of the PIN diode reported by Taniyasu et al. was $0.02 \mu \mathrm{W}$ at $40 \mathrm{~mA}$. The measured external quantum efficiency (ca. 6-10\%) was far from the value of $20 \%$ of commercial UVC LEDs. [19]

The biggest problem faced by nitride-based UVC and UVB LEDs is the application of AIN with a high Al content, because the doping activation energy of $\mathrm{Mg}$ is very high and the doping efficiency is very low. The thickness of p-AlGaN must, therefore, be increased to satisfy the requirements of quasi-charge neutrality. However, up to now, the conductivity of the neutral region of AIGaN DUV still cannot meet the condition of quasi- charge neutrality when injected into the quantum well (excess electrons are injected and then the same number of holes are injected). It will need a longer thermal equilibrium reaction time. And also make the series resistance larger, resulting in bias loss. [20, 21] In addition, because of the effects of light absorption and the series resistance, the thickness of the p-GaN contact layer cannot be greater than $40 \mathrm{~nm}$. Therefore, the mismatch in the degrees of electron/hole injection in the active layer of the quantum well will lead to a lack of quasi-charge neutrality. In addition, the traditional UVB structure Loading [MathJax]/jax/output/CommonHTML/fonts/TeX/fontdata.js e quantum well active layer, but it blocks not 
only the overflow of electrons but also the injection of holes. The presence of exciton emission can greatly improve the luminous efficiency of an LED device. The exciton binding energies of AIN, diamond, and $\mathrm{ZnO}$ are 80,80 , and $60 \mathrm{meV}$, respectively, but at present it remains difficult to form quality $p-n$ junctions from these three materials. AIGaN is the most promising material for forming $p-n$ junctions to exploit exciton emissions. Furthermore, the AIN template and sapphire have been the best substrates for UVC and UVB emissions. In addition to the doping activation energy, limitations in heterojunction lattice mismatch epitaxial growth technology have made it more difficult to improve the luminous efficiency in the UVB band than in the UVC band. Therefore, to ensure the exciton emission of nitride-based compounds in the UVB band at room temperature, it will be necessary to shorten the relaxation time of the holes in the neutral region and allow the luminescent layer to reach a state of quasi-charge neutrality. Therefore, by considering the requirements for light absorption and the relaxation time of the holes, we designed an LED containing a 20-nm-thick layer of p-GaN. In addition, before depositing the p-GaN layer, we abandoned the AIGaN EBL and replaced it with a two-fold p-Al 0.55 GaN/p-Al ${ }_{0.4} \mathrm{GaN}$ structure to improve the crystal quality. After depositing the two-fold layer structure, to minimize the two-dimensional electron gas (2DEG) produced by the spontaneous polarization and piezoelectric polarization between materials, which would lead to electron/hole recombination and parasitic luminescence in the neutral region of the p-type structure, we also deposited a 50-nm-thick p-AlGaN-to-p-GaN grading composition layer. Finally, we deposited the 20-nm-thick p-GaN layer to ensure a sufficient number of holes and good ohmic contact. If too many electrons or holes accumulated near the lowest energy of the quantum well, we suspected that a screening effect would break apart the formed exciton pairs. Because exciton emission is generally observed from relatively pure materials, but not from highly doped materials, we did not dope the quantum wells or barriers. Using this design and arrangement, we observed exciton emission behavior from AlGaN-based LEDs. Imagine we were matchmakers trying to combine Mr. Electrons and Miss. Holes

to ensure happy marriages, is there any possible approach? With the semiconductor exciton light-emitting device, the answer is affirmative. Because of the Coulomb attraction, the energy of the electron-hole pair as a whole has increased. Additionally, since Electron and Holes are close to each other and with the same velocity, the rate of direct recombination will be faster, improving the efficiency by the exciton emission. In this paper, AIGaN UVB exciton emission by increasing the numbers of Holes. Also, we make some energy sacrifices, photon absorbed, in exchange for the Global green technology.

\section{Experimental Methods}

The epitaxial growth of AIGaN-based LEDs was performed using low-pressure metal-organic chemical vapor deposition (LP-MOCVD). Trimethylaluminum, trimethylgallium, silane, and ammonia were used as $\mathrm{Al}, \mathrm{Ga}, \mathrm{Si}$, and $\mathrm{N}$ sources, respectively.

To obtain the UVB LED (sample A), a 2.2- $\mu$ m-thick AIN buffer layer was first grown on a 2-inch (0001) oriented sapphire substrate. Next, an interlayer consisting of 30 periods of a $2.5-\mathrm{nm}$ AlN/17.5-nm AlGaN superlattice with an equivalent Al composition (0.72) was grown on the AIN buffer layer. A 1.5- $\mu$ m-thick undoped $\mathrm{Al}_{n}{ }_{6} \mathrm{Ga}_{n} \mathrm{~N}$ layer was the grown on the superlattice interlayer. Subsequently, a $2-\mu \mathrm{m}$-thick SiLoading [MathJax]/jax/output/CommonHTML/fonts/TeX/fontdata.js 
doped $\mathrm{n}-\mathrm{Al}_{0.5} \mathrm{Ga}_{0.5} \mathrm{~N}$ layer was grown as the $\mathrm{n}$-contact. The active region included four periods of $\mathrm{Al}_{0.35} \mathrm{Ga}_{0.65} \mathrm{~N}(2 \mathrm{~nm}) / \mathrm{Al}_{0.45} \mathrm{Ga}_{0.55} \mathrm{~N}(8 \mathrm{~nm})$ multiple quantum wells (MQWs). A two-fold Mg-doped p$\mathrm{Al}_{0.55} \mathrm{Ga}_{0.45} \mathrm{~N}(10 \mathrm{~nm}) / \mathrm{Mg}$-doped p-Al $\mathrm{O}_{0.4} \mathrm{Ga}_{0.6} \mathrm{~N}(2 \mathrm{~nm})$ structure, replacing the conventional AIGaN EBL, was grown, followed by a 50-nm-thick Mg-doped p-Al $\mathrm{A}_{0.3} \mathrm{Ga}_{0.7} \mathrm{~N}$-to- $\mathrm{p}-\mathrm{GaN}$ grading layer. Finally, a 20-nmthick Mg-doped p-GaN layer was deposited to serve as the p-contact layer and also to modulate the hole relaxation time (Fig. 1).

To obtain the UVC LED (sample B), the AIN buffer layer, 30-period superlattice, and undoped $\mathrm{Al}_{0.6} \mathrm{Ga}_{0.4} \mathrm{~N}$ layer were prepared in the same manner as those in sample A. Subsequently, a 2- $\mu \mathrm{m}$-thick Si-doped $\mathrm{n}$ $\mathrm{Al}_{0.6} \mathrm{Ga}_{0.4} \mathrm{~N}$ layer was grown as the $\mathrm{n}$-contact. The active region included four periods of $\mathrm{Al}_{0.45} \mathrm{Ga}_{0.55} \mathrm{~N}(2$ $\mathrm{nm}) / \mathrm{Al}_{0.55} \mathrm{Ga}_{0.45} \mathrm{~N}(8 \mathrm{~nm}) \mathrm{MQWs}$. A two-fold Mg-doped p-Al ${ }_{0.55} \mathrm{Ga}_{0.45} \mathrm{~N}(10 \mathrm{~nm}) / \mathrm{Mg}$-doped p$\mathrm{Al}_{0.45} \mathrm{Ga}_{0.55} \mathrm{~N}(2 \mathrm{~nm})$ structure was then grown, replacing the conventional AIGaN EBL, followed by a 50 nm-thick Mg-doped p-Al ${ }_{0.3} \mathrm{Ga}_{0.7} \mathrm{~N}$-to-p-GaN grading layer. Finally, a 20-nm-thick Mg-doped p-GaN layer was deposited to serve as the p-contact layer and also to modulate the hole relaxation time.

After performing the LP-MOCVD growth processes, the samples were annealed in a $\mathrm{N}_{2}$ ambient to activate the Mg-dopants. The LED chips were fabricated using standard chip-processing technologies. Mesa structures were defined through inductively coupled plasma etching to expose the $\mathrm{n}-\mathrm{Al}_{0.5} \mathrm{Ga}_{0.5} \mathrm{~N}$ layer surface. $\mathrm{N}$-contacts of $\mathrm{Ti} / \mathrm{Al} / \mathrm{Ti} / \mathrm{Au}(100 / 200 / 30 / 100 \mathrm{~nm})$ were deposited through electron-beam evaporation and annealed through rapid thermal annealing at $980^{\circ} \mathrm{C}$ for $60 \mathrm{~s}$. To form the transparent $\mathrm{p}$ contact, a 50-nm-thick layer of indium tin oxide (ITO) was sputter-deposited and annealed at $600^{\circ} \mathrm{C}$ for 10 min. The LED chips were completed with the deposition of Ti/Pt/Au (50/30/100 nm) and AuSn $(3 \mu \mathrm{m})$; the p-contact area was approximately $0.14 \mathrm{~mm}^{2}$.

The prepared flip-chips were bonded on an AIN direct plating ceramic lead frame using the eutectic method (AD211 plus, ASM); a covering quartz glass served as an optical lens. The packaged samples were soldered onto an Al metal core printed circuit board. The light output power (LOP), the currentvoltage characteristics, and the electroluminescence (EL) spectrum of the samples were measured using an ATA-5000 LED photoelectric measurement system (Everfine) equipped with a 30-cm-diameter integrating sphere. During measurement, the temperature of the heat-sink mounting the packaged sample was controlled between 298 and $368 \mathrm{~K}$ at a driving direct current was $40 \mathrm{~mA}$ (current density: ca. $28.5 \mathrm{~A}$ $\mathrm{cm}^{-2}$ ). To minimize the effect of self-heating of the chips, the stop interval was set to 3 min during each continuous wave $(\mathrm{CW})$ measurement.

\section{Results And Discussion}

Figures 2(a) and 2(b) present the EL band peak positions (measured at room temperature) of the two samples before and after performing burning tests at $40 \mathrm{~mA}$. Sample A exhibited a clear exciton emission, with its peak near $306 \mathrm{~nm}$, and a band-to-band emission at $303 \mathrm{~nm}$. The peak at $306 \mathrm{~nm}$ also minht hn thn aunntum wall anmmonitinn that mawnm thn gpatial potential fluctuations, but we observed 
that narrow band full widths at half maximum (FWHM) less than $10 \mathrm{~nm}$ and the composition fluctuations will result in a larger FWHM, so we prefer this peak was caused by exciton emission. Because the excitons existed in the crystal and not in free space, the binding energy was decreased by the reduced mass and the dielectric constant, where $R_{\mathrm{y}}$ is equal to $13.6 \mathrm{eV}$ and $n$ is an integer greater than or equal to 1.

$$
E_{X}=-\left(\frac{m_{\mathrm{r}} q^{4}}{2 h^{2} \epsilon^{2}}\right) \frac{1}{n^{2}}=-\frac{\left(m_{\mathrm{r}} / m_{0}\right)}{\epsilon_{r}^{2}} \frac{R_{y}}{n^{2}}
$$

1

After applying Eq. (1), we obtained an exciton bonding energy for $\mathrm{Al}_{0.35} \mathrm{Ga}_{0.65} \mathrm{~N}$ of approximately $39 \mathrm{meV}$. Accordingly, we confirmed that the signal near $306 \mathrm{~nm}$ arose from exciton emission. Sample B provided only a Gaussian peak emission at a wavelength of approximately $272 \mathrm{~nm}$. We calculated the Al content in the AlGaN structure by using the Schrödinger wave equation with a quantized level shift and following Vegard's law; the $\mathrm{Al}_{0.45} \mathrm{Ga}_{0.55} \mathrm{~N}$ band-to-band emission was approximately $270 \mathrm{~nm}$. Therefore, we attributed the emission at $272 \mathrm{~nm}$ mainly to the band-to-band transition. For both samples, increasing the temperature caused the intensity of the band-to-band emission to decrease and shift to slightly longer wavelength. The relative intensity of the exciton emission in sample A decreased as a result of a thermal energy effect. Sample A provided the exciton emission at temperatures of up to $363 \mathrm{~K}$. Several reports have described parasitic peaks extending from the low-energy side of the main peak for UVC LEDs. [22] These parasitic peaks are presumably associated with recombination occurring through deep level defects and electron overflow and through polarization doping recombination in the p-AIGaN neutral region. In this present study, we did not observe any obvious parasitic peaks from sample $B$ at any temperature up to $363 \mathrm{~K}$. Therefore, we suggest that defect-related recombination did not occur in sample $B$ under the injection conditions in the temperature range from approximately 298 to $363 \mathrm{~K}$. Accordingly, using the two-fold $\mathrm{p}-\mathrm{Al}_{0.55} \mathrm{GaN} / \mathrm{p}-\mathrm{Al}_{0.4} \mathrm{GaN}$ structure (to improve the crystal quality), the 50 -nm-thick pAlGaN-to-p-GaN grading composition layer, and the 20-nm-thick p-GaN layer (to ensure a sufficient number of holes and good ohmic contact) effectively depressed the emission band arising from defects or impurities. Santi et al. reported [23] that the normalized intensity of parasitic peak 3 (ca. $340 \mathrm{~nm}$ ) from a UVB LED decreased monotonically upon increasing the temperature from 100 to $400 \mathrm{~K}$; they suggested that the peak 3 originated from radiative transitions through deep levels in the quantum barrier next to the $E B L$, because of its broad shape.

Figures 3(a) and 3(b) reveal the temperature-dependence of the peak wavelengths of samples $A$ and $B$ at $40 \mathrm{~mA}$. The peak wavelengths and full widths at half maximum (FWHMs) of both samples shifted slightly-from $306.0 \mathrm{~nm}(9.612 \mathrm{~nm})$ to $306.6 \mathrm{~nm}(11.02 \mathrm{~nm})$ and from $271.8 \mathrm{~nm}(10.34 \mathrm{~nm})$ to $272.5 \mathrm{~nm}$ $(11.24 \mathrm{~nm})$, respectively-upon increasing the temperature from 298 to $363 \mathrm{~K}$. Consistent with Varshni's law, the red-shifts were caused by narrowing of the band gap upon increasing the temperature. The Loading [MathJax]/jax/output/CommonHTML/fonts/TeX/fontdata.js ation of the reciprocal of $E_{g}(T)$, because of 
poorer quality resulting from the higher Al content and more alloy scattering layer in the AIGaN MQW. Furthermore, the density of carriers available for radiative recombination inside in MQWs had decreased.

Sample A was more likely to experience a decrease in the radiation recombination rate and an increase in the non-radiative recombination rate upon increasing the temperature. At temperatures of up to $363 \mathrm{~K}$, the emission of sample A appeared as two Gaussian peaks, the exciton emission and band-to-band emission.

To obtain a deeper understanding of the temperature-dependence of the emissions from the UVB and UVC LEDs, we measured the non-normalized light output power of samples $A$ and $B$ at various temperatures (Fig. 4). Upon increasing the temperature, the light output power from both samples decreased monotonically, but the slope of the line for sample A was much smaller than that for sample B. The exciton emission from sample A was less likely to be scattered by phonons. The photonic device temperature reliability is known to be affected by the carrier confinement factor. A larger discontinuity in the conductive band enhances the thermal stability. Because the values of $\Delta E_{c}$ of samples $A$ and $B$ were nearly identical, the electroluminescence of sample A must have arisen, in part, from the exciton emission. According to the fitting data derived from the temperature-dependence of the light output power, the values of characteristic temperature $\left(T_{0}\right)$ of samples $A$ and $B$ were 328 and $108 \mathrm{~K}$, respectively. Chhajed et al. reported that a larger value of $T_{0}$ could decrease the contribution of non-radiative recombination to the total carrier recombination, due to saturation of the non-radiative recombination paths; as a result, the activation energy of the non-radiative recombination centers and the energy required to overcome the confining potentials would both increase. [24]

Ploch et al. [25] investigated the influence of the dislocation density and the barrier height on the temperature-stability of the light output power from a 380-nm LED. The decrease in the temperaturestability was more pronounced in a sample with high TDD (threading dislocation density) and weakly influenced by the barrier height when the current density was less than $50 \mathrm{~A} \mathrm{~cm}^{-2}$. In this present study, both of our samples had the same structure below the Si-doped n-contact layer, and no indium was present in the MQW active region. Nevertheless, sample A was less temperature-sensitive than sample $B$ in its light output power at a current density of approximately $28.5 \mathrm{~A} \mathrm{~cm}^{-2}$. Hence, we attribute the improved temperature-stability of sample A to its lower effective TDD and superior carrier confinement in the active region, due to the exciton quantum confine effect. The behavior of sample A can be explained by considering that its exciton emission was less likely to be scattered by lattice vibrations to form free charge carriers, and by its insensitivity to impurities or defects. As mentioned above, limitations in heterojunction lattice mismatch epitaxial growth technology have made it more difficult to improve the luminous efficiency of the UVB band relative to the UVC band. As a result, the TDD in sample A had decreased effectively.

\section{Conclusion}


Among the semiconductor materials, two light-emitting materials are considered to have great potentialAIN and $\mathrm{ZnO}$, but each of them has very low p-type doping efficiency and are difficult to prepare. In this paper, we present a new approach toward a nitride-based UVB LED that successfully realizes an exciton emission in the UVB band. Our design suppressed the parasitic emission caused by electron overflow in a typical UVB. In addition, the p-type layer structure and doping profile ensured conditions of quasi-charge neutrality in the UVB quantum well. Despite the content of $\mathrm{Al}$ in the luminescent layer of sample $A$ being smaller than that of sample $B$, sample $A$ was less temperature-sensitive in its the light output power at a current density of approximately $28.5 \mathrm{~A} \mathrm{~cm}^{-2}$. According to the fitting data derived from the temperaturedependence of the light output power, the characteristic temperatures $\left(T_{0}\right)$ of samples $A$ and $B$ were 328 and $108 \mathrm{~K}$, respectively.

\section{Abbreviations}

UVB LED: Ultraviolet-B light-emitting diodes; UVC LED: Ultraviolet-C light-emitting diodes; 2DEG: Twodimensional electron gas; LP-MOCVD: Low-pressure metal-organic chemical vapor deposition; LOP: Light output power; CW: Continuous wave; FWHM: Full widths at half maximum;

\section{Declarations}

\section{Competing interests}

The authors declare that they have no competing interests.

\section{Funding}

This study was supported in part by the Ministry of Science and Technology (MOST), Taiwan, under contract MOST 109-2221-E-182 -060, and in part by the Chang Gung Memorial Hospital, under grant BMRP 591.

\section{Authors' contributions}

M.J. Lai, R.S. Liu designed the experiments. R.M. Lin and C.H. Kuan analyzed data. T.Y. Liu and S.M. Huang discussed the results and contributed to the writing of the manuscript. All authors read and approved the fnal manuscript.

\section{Available of data and materials}

The datasets supporting the conclusions of this article are available in the article 


\section{Acknowledgment}

We thank Professor Keh-Yung Cheng for helpful discussions and Dr. Shu-chang Wang (School of Electronic and Information Engineering, Changshu Institute of Technology, 215500 Jiangsu, P. R. China) for measurement of the temperature-dependence EL.

\section{References}

[1] Mike Cooke. Excitonic prospects for UV. III-Vs Review. 2006; doi: 10.1016/S0961-1290(06)71711-2

[2] Michael Kneissl, Tae-Yeon Seong, Jung Han and Hiroshi Amano. The emergence and prospects of deep-ultraviolet light-emitting diode technologies. Nature Photonics. 2019; doi: 10.1038/s41566-0190359-9

[3] Godar DE, Miller SA and Thomas DP. Immediate and delayed apoptotic cell death mechanisms: UVA versus UVB and UVC radiation. Cell Death and Differentiation. 1994; doi: 10.1038/cdd.2008.157

[4] Haitao Chen, Yutao Tang, Tian Jiang and Guixin Li. Nonlinear Nanophotonics With 2D Transition Metal Dichalcogenides. Comprehensive Nanoscience and Nanotechnology. 2nd ed. Elsevier, 2019, pp. 305-318.

[5] Kin Fai Mak, Changgu Lee, James Hone, Jie Shan, and Tony F. Heinz. Atomically thin $\mathrm{MoS}_{2}$ : A new direct-gap semiconductor. Physical Review Letters. 2010; doi: 10.1103/PhysRevLett.105.136805

[6] Andrea Splendiani, Liang Sun, Yuanbo Zhang, Tianshu Li, Jonghwan Kim, Chi-Yung Chim, Giulia Galli, and Feng Wang. Emerging photoluminescence in monolayer MoS $_{2}$. Nano Letter. 2010; doi:

$10.1021 / \mathrm{nl} 903868 \mathrm{w}$

[7] Keh-Yung Cheng. III-V Compound Semiconductors and Devices. Springer; 2020.

[8] Di Xiao, Gui-Bin Liu, Wanxiang Feng, Xiaodong Xu, and Wang Yao. Coupled spin and valley physics in monolayers of $\mathrm{MoS}_{2}$ and other group-VI dichalcogenides. Physical Review Letters. 2012; doi:

10.1103/PhysRevLett.108.196802

[9] Xiaodong Xu, Wang Yao, Di Xiao and Tony F. Heinz. Spin and pseudospins in layered transition metal dichalcogenides. Nature Physics. 2014; doi: 10.1038/nphys2942

[10] Haug, H. and Koch, S.W. Quantum Theory of the Optical and Electronic Properties of Semiconductors. World Scientific Publishing; 2009.

[11] Kin Fai Mak and Jie Shan. Photonics and optoelectronics of 2D semiconductor transition metal dichalcogenides. Nature Photonics. 2016; doi: 10.1038/nphoton.2015.282 
[12] J. Feldmann, G. Peter, E. O. Göbel, P. Dawson, K. Moore, C. Foxon, and R. J. Elliott. Linewidth dependence of radiative exciton lifetimes in quantum wells. Physical Review Letters. 1987; doi: 10.1103/PhysRevLett.59.2337

[13] Hartmut Haug and L. Banyai. Optical Switching in Low-Dimensional Systems. Springer; 2012.

[14] K. Sivalertporn, L. Mouchliadis, A. L. Ivanov, R. Philp, and E. A. Muljarov. Direct and indirect excitons in semiconductor coupled quantum wells in an applied electric field. Physical Review B. 2012; doi: 10.1103/PhysRevB.85.045207

[15] Satoshi Koizumi, Kenji Watanabe, Masataka Hasegawa and Hisao Kanda Ultraviolet emission from a diamond pn junction. Science. 2001; doi: 10.1002/chin.200140017

[16] T. Makino, Y. Segawa, M. Kawasaki and H. Koinuma. Optical properties of excitons in ZnO-based quantum well heterostructures. Semiconductor Science and Technology. 2004; doi: 10.1088/0268$1242 / 20 / 4 / 010$

[17] Yoshitaka Taniyasu, Makoto Kasu and Toshiki Makimoto. An aluminium nitride light-emitting diode with a wavelength of 210 nanometres. Nature. 2006; doi: 10.1038/nature04760

[18] John Simon, Vladimir Protasenko, Chuanxin Lian, Huili Xing and Debdeep Jena. Polarization-induced hole doping in wide-band-gap uniaxial semiconductor heterostructures. Science. 2010; doi:

10.1126/science. 1183226

[19] J. Thirumalai. Light-Emitting Diode. IntechOpen; 2018.

[20] M. Ajmal Khan, Eriko Matsuura, Yukio Kashima and Hideki Hirayama. Overcoming the current injection issue in the $310 \mathrm{~nm}$ band AIGaN UVB light-emitting diode. Japanese Journal of Applied Physics. 2019; doi: 10.7567/1347-4065/ab460b

[21] M. Ajmal Khan, Yuri Itokazu, Noritoshi Maeda, Masafumi Jo, Yoichi Yamada and Hideki Hirayama. External quantum efficiency of $6.5 \%$ at $300 \mathrm{~nm}$ emission and $4.7 \%$ at $310 \mathrm{~nm}$ emission on bare wafer of AIGaN-based UVB LEDs. ACS Applied Electronic Materials. 2020; doi: 10.1021/acsaelm.0c00172

[22] Tingzhu Wu, Yue Lin, Zhangbao Peng, Huashan Chen, Zhibin Shangguan, Meng Liu, Sung-Wen Huang Chen, Chih-Hao Lin, Hao-Chung Kuo, and Zhong Chen. Interplay of carriers and deep-level recombination centers of 275-nm light-emitting diodes: Analysis on the parasitic peaks over wide ranges of temperature and injection density. Optics Express. 2019; doi: 10.1364/OE.27.0A1060

[23] Carlo De Santi, Matteo Meneghini, Desiree Monti, Johannes Glaab, Martin Guttmann, Jens Rass, Sven Einfeldt, Frank Mehnke, Johannes Enslin, Tim Wernicke, Michael Kneissl, Gaudenzio Meneghesso, and Enrico Zanoni. Recombination mechanisms and thermal droop in AIGaN-based UV-B LEDs.

Photonics Research. 2017; doi: 10.1364/PRJ.5.000A44

Loading [MathJax]/jax/output/CommonHTML/fonts/TeX/fontdata.js

Page 10/13 
[24] X.A. Cao, S.F. LeBoeuf and T.E. Stecher. Temperature-dependent electroluminescence of AlGaN-based UV LEDs. IEEE Electron Device Letters. 2006; doi: 10.1109/LED.2006.873763

[25] N Lobo Ploch, S Einfeldt, M Frentrup, J Rass, T Wernicke, A Knauer, V Kueller, M Weyers and M Kneissl. Investigation of the temperature dependent efficiency droop in UV LEDs. Semiconductor Science and Technology. 2013; doi: 10.1088/0268-1242/28/12/125021

\section{Figures}

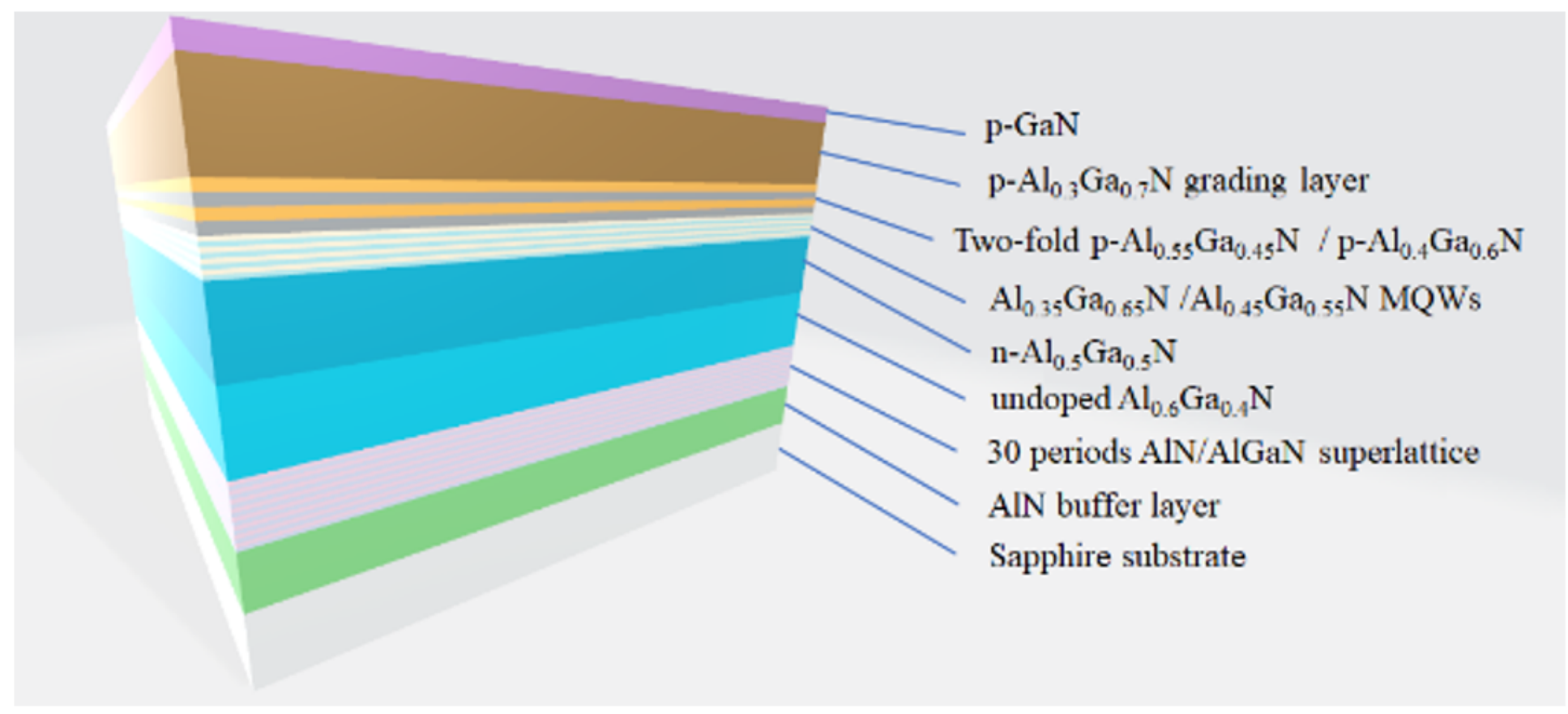

\section{Figure 1}

Schematic representation of the epitaxial structure of sample A. 


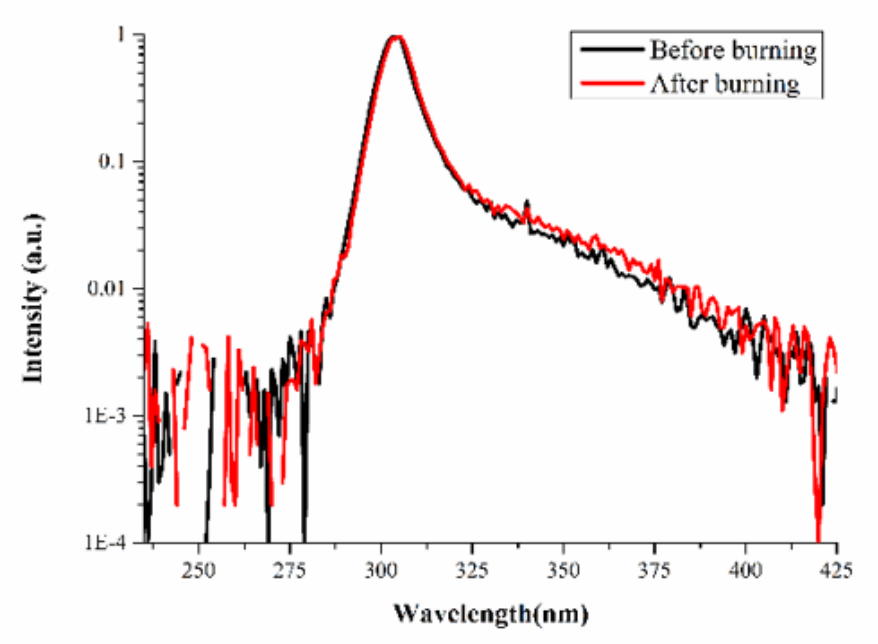

(a)

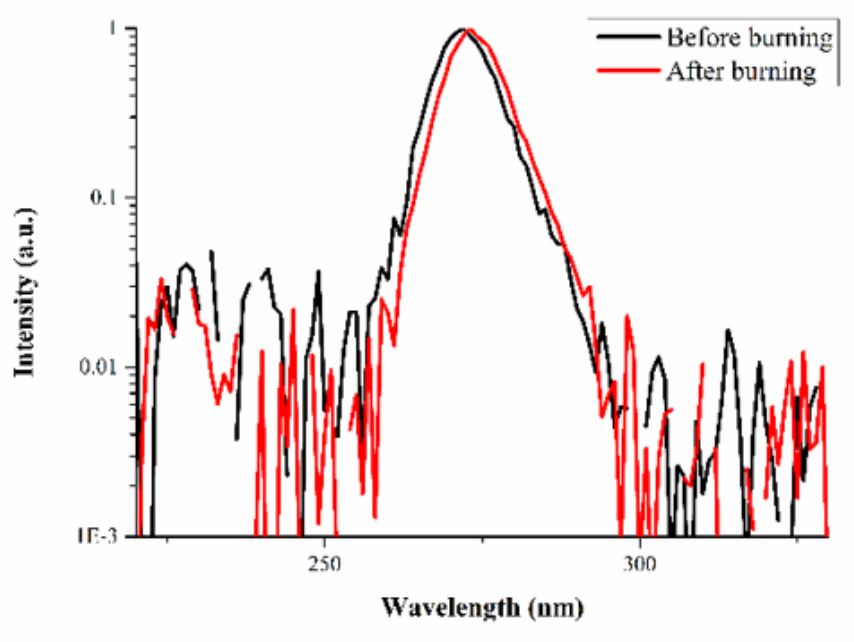

(b)

\section{Figure 2}

EL band peak positions, measured at room temperature before and after burning tests, of (a) sample $A$ $(\mathrm{Vf}=5.246 \mathrm{~V} @ 40 \mathrm{~mA})$ and $(\mathrm{b})$ sample B ( $\mathrm{Vf}=5.530 \mathrm{~V} @ 40 \mathrm{~mA})$.

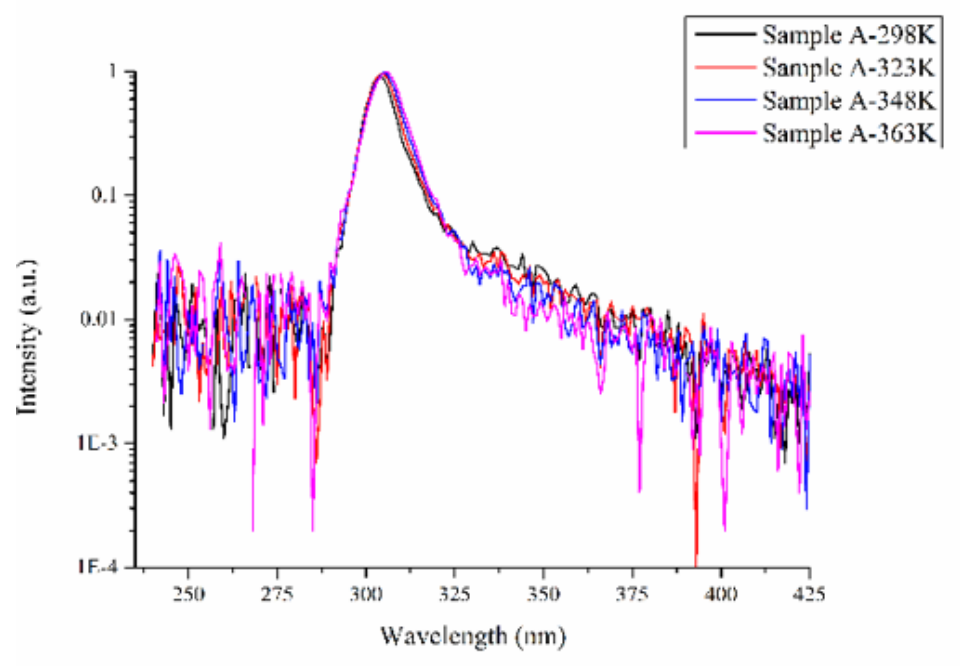

(b)

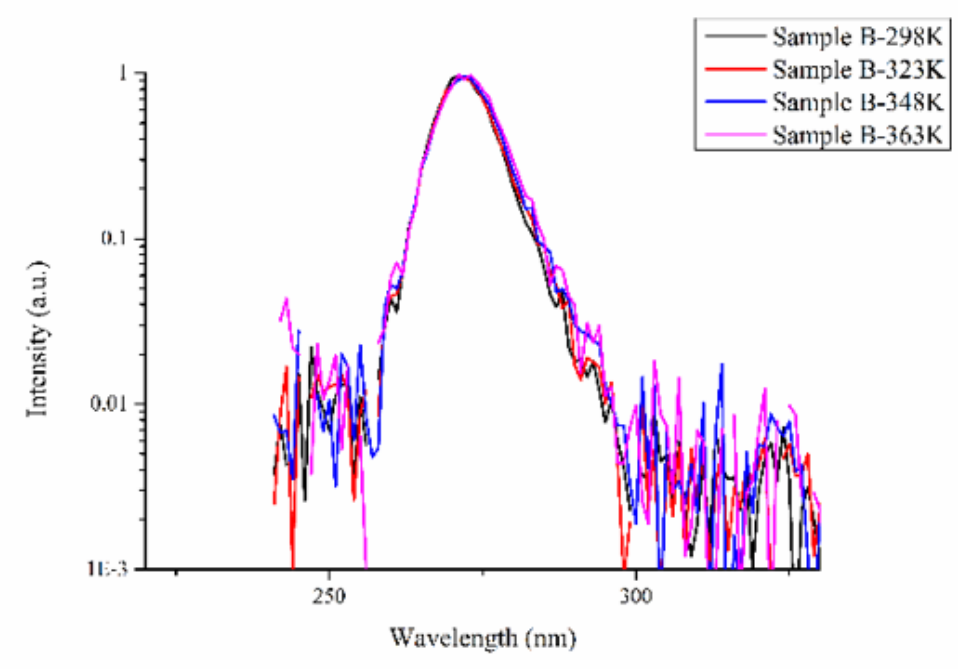

(b)

Figure 3

Temperature-dependence of the EL peak wavelengths of (a) sample A ( Vf = 5.246 V @40 mA) and (b) sample B (Vf=5.530 V @40 mA). 


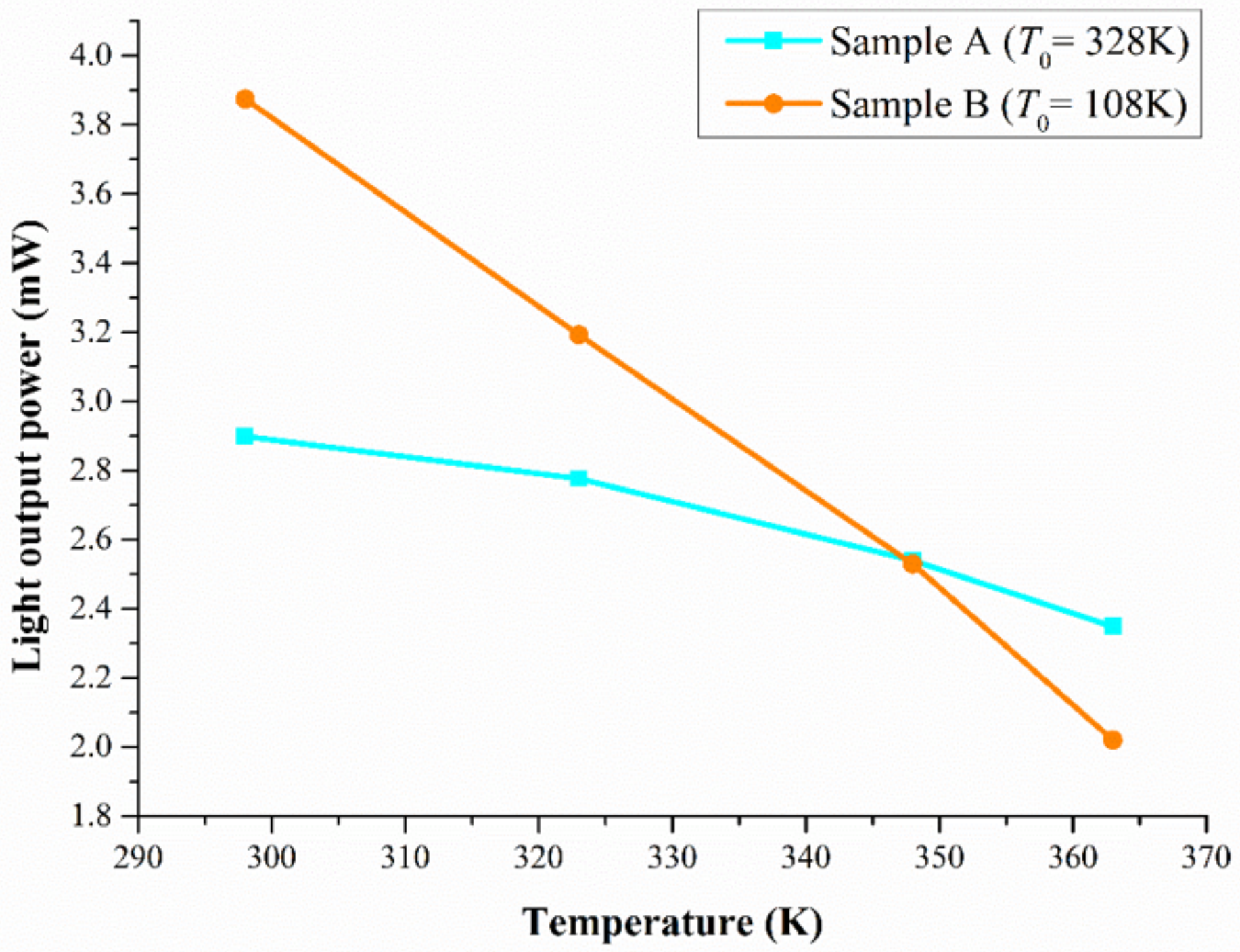

Figure 4

Light output power from samples $A$ and $B$ at various temperatures at $40 \mathrm{~mA}$. 